\title{
Efeito de desordem energética na dinâmica de éxcitons em cristais de tetraceno
}

Marina Pinheiro Dourado ${ }^{1^{*}}$ (dourado.marina@hotmail.com) (PG), Demétrio Antônio da Silva Filho ${ }^{1}(\mathrm{PQ})$, Leonardo Evaristo de Sousa $^{1}(\mathrm{PG})$, Pedro Henrique de Oliveira Neto ${ }^{1}$ (PQ).

1Instituto de Física, Universidade de Brasília, 70919-970, Brasília, Brasil.

Palavras-chave: tetraceno, espectro de emissão, desordem.

\section{Introdução}

A eficiência de dispositivos tais como células fotovoltaicas e $L E D$ s está relacionada à dinâmica de éxcitons dentro do material. Mesmo sendo estudado teoricamente durante décadas, a observação de difusão desses e dos efeitos de desordem na transferência de energia ainda são recentes ${ }^{[1]}$. Akselrod et al. verificaram dois tipos preferenciais de transporte dessas quase-partículas em cristais moleculares de tetraceno. Além disso, o estudo mostra, através dos espectros de absorção e emissão, como a transferência de energia é impedida por efeitos de desordem. Neste trabalho, estudamos a desordem energética provocada pela atenuação no espectro de emissão de cristais de tetraceno.

\section{Metodologia}

Na primeira etapa do estudo, calculamos a geometria de equilíbrio do tetraceno, tanto para o estado fundamental (S0), quanto para o primeiro estado excitado (S1). Para isso, foram realizados cálculos, no programa Gaussian $09^{[2]}$, de otimização e frequência para cada estado usando os funcionais B3LYP, M062X e WB97XD dentro da teoria do funcional densidade com a base 6-31G $(\mathrm{d}, \mathrm{p})$. Uma vez encontradas as geometrias de equilíbrio, obtivemos os espectros de emissão e absorção através do software Newton$X^{[3]}$.

\section{Resultados e Discussão}

Em cristais de tetraceno, a transferência de energia é do tipo Förster. Isso foi verificado devido à presença de sobreposição dos espectros de absorção e emissão. Como esse mecanismo é dado por interação dipolo-dipolo entre moléculas a uma distância (raio de Förster) ${ }^{[4]}$, calculamos o momento de dipolo, o qual está associado ao fator de orientação do cristal, e o raio de Förster, através dos espectros.

$\mathrm{O}$ raio de Förster foi calculado por meio da equação abaixo, em que c é a velocidade da luz; $k$, um fator que varia de 0 a 2 e está relacionado com a orientação entre dipolos; $n$, índice de refração do meio e $\omega$, frequência. A integral foi avaliada numericamente na região de overlap entre os espectros de emissão e absorção $I_{D}(\omega)$ e $\alpha_{A}(\omega)$ respectivamente.

$$
R_{F}{ }^{6}=\tau_{D}^{0} \frac{9 c^{4} k^{2}}{8 \pi n} \int_{0}^{\infty} \frac{d \omega}{\omega^{4}} I_{D}(\omega) \alpha_{A}(\omega)
$$


Posteriormente, simulamos pela atenuação do espectro de emissão e verificamos a sua influência no raio de Förster. Por fim, o estudo acerca da dinâmica é feito pelo Monte Carlo cinético, onde encontramos o comprimento de difusão do éxciton e a difusividade.

\section{Conclusão}

Através de atenuação do espectro de emissão de cristais de tetraceno, observamos como a dinâmica de éxcitons nesse material é influenciada pelos efeitos de desordem energética. Além disso, obtemos dados que permitem compreender o funcionamento de materiais com mecanismo de transferência de Förster.

\section{Agradecimentos}

Os autores agradecem à CAPES, à FAP-DF e ao CNPq o apoio financeiro.

\section{Referências}

[1] AKSELROD, G. M.; et al. Visualization of exciton transport in ordered and disordered molecular solids. Nature Communications, volume 5, 2014.

[2] FRISCH, M.J.; et al. Gaussian 09, Revision A.02. In., Series Gaussian, Inc., Wallingford CT; 2009

[3] BARBATTI, M; et al. Wiley Interdiscip. Rev.: Comput. Mol. Sci. 4, 26; 2014.

[4] DE PAULI, M. . Fenômenos de interfaces e aplicações em sistemas orgânicos. Tese de Doutorado. Universidade Federal de Minas Gerais, 2016. 